\title{
Factors Associated with Potentially Inappropriate Medication Use in Older Adults in China applying 2019 Updated Beers Criteria
}

\author{
Ming-ming $\mathrm{Yan}^{1}$, Shu-Shan $\mathrm{Wu}^{2}$, Jing $\mathrm{Li}^{2}$, Nang $\mathrm{Lu}^{2}$, Xiao-Yan Qiu ${ }^{2}$, and Ming-Kang \\ Zhong $^{2}$
}

${ }^{1}$ Huashan Hospital Fudan University

${ }^{2}$ Affiliation not available

May 29, 2020

\begin{abstract}
Objectives This study aimed to measure the prevalence of potentially inappropriate medications (PIMs) in Chinese elderly population according to the 2019 Beers Criteria and to evaluate the associative factors of PIMs. Methods Prescriptions of patients aged over 65 years who were hospitalized during January 2017 to December 2017 were retrieved from Hospital Prescription Analysis Cooperation Project, covering seventy-six facilities in six cities in China. PIMs were identified by 2019 Beers Criteria. Results In total, $40.1 \%$ elderly patients were treated with at least one PIM independent of their diagnoses or conditions according to the 2019 Beers Criteria. The most frequently prescribed inappropriate medications independent of diagnoses or conditions were proton-pump inhibitors (12.9\%). Diuretics (8.2\%) and central nervous system medications (6.9\%) also showed a relatively high prevalence among the investigated patients. Based on the Beers Criteria's lists of drug-disease interactions, elderly patients with central nervous system conditions (including delirium, dementia or cognitive impairment) or with a history of falls or fractures were most commonly exposed to PIMs. Logistic regression revealed that inappropriate medication use was associated with age ([?]80 years old), number of conditions ([?]5), number of medications prescribed ([?]10) and class of healthcare facilities. Conclusion This study was able to confirm that the phenomenon of prescribing potentially inappropriate medications was common in China. Our findings support the importance of developing explicit criteria to detect PIMs in China and implementing effective interventions to promote better quality of prescribing for elderly patients.
\end{abstract}

\section{INTRODUCTION}

Elderly people are regarded as a special group of the whole society population, so every aspect of their life should be given specific attention. Due to the decline of body's functions, elderly people generally experience more chronic conditions or diseases, which usually require co-administration of multiple medications and make them more vulnerable to adverse drug events or medication-related problems.

The Beers Criteria, originated in 1991, were the first generally accepted explicit criteria in aid of better prescribing for elderly patients and have been used widely. It was first developed for nursing home patients. Under the support of the American Geriatrics Society (AGS), five versions of Beers Criteria have been compiled and updated, respectively in 1997, 2003, 2012, 2015, and 2019, and are now intended for application and evaluation medications in patients over the age of $65 .{ }^{1-6}$ Beers criteria have listed, even though not every, potentially inappropriate medications (PIMs), namely medications with adverse risks outweighing benefits when compared to other alternative therapies. ${ }^{7}$

There are a number of elderly patients in developing countries and regions who are exposed to inappropriate prescriptions or polypharmacy, and this significantly contributes to global PIMs. ${ }^{8}{ }^{9} \mathrm{PIMs}$ have been proved to be related to poor health outcomes, including falls, confusion, and even mortality, leading to negative clinical and economic consequences. ${ }^{10,11}$ It has also been widely believed that PIMs are preventable. ${ }^{12-14}$ Interventions 
applying explicit criteria have been found to be an effective strategy to reduce inappropriate medication usage. $^{4,15,16}$

The aim of this research was to estimate the prevalence of PIMs in elderly patients in China based on 2019 updated Beers Criteria. The relationship between the prevalence of PIMs and four main factors including age, number of current diseases, number of prescribed medications, and hospital tier was also investigated. The results can serve as a baseline for future studies if the Beers Criteria were to be generally used in China.

\section{METHODS}

\section{Prescription data and sample selection}

This retrospective study focused on elderly patients who were hospitalized from January 2017 to December 2017 in seventy-six hospitals in China. Elderly patients were defined as patients over 65 years of age before or during their hospitalization.

Prescription data were obtained from Hospital Prescription Analysis Cooperation Project. This project was launched in 1997 by a renowned pharmacist in China named Dakui Li. It allowed for the creation of a database that contains anonymized prescription information from seventy-six hospitals located in six cities in China: Beijing, Tianjin, Shanghai, Hangzhou, Guangzhou, and Chengdu. The participating hospitals must follow the 'recording guidelines' that require them to strictly control and monitor their own prescription information and each participating hospital has open access to the prescription information database. So far, there is considerable amount of patient prescription information in the database, allowing us to retrospectively evaluate the inappropriate pharmaceutical treatments. ${ }^{17}$

\section{Data collection and evaluation procedures}

For all patients aged over 65 years old, demographic information (age and gender), clinical history (diseases and comorbidities) and prescription information were included. Age groups were divided into seven 5-year age groups (66-70, 71-75, 76-80, 81-85, 86-90, 91-95 and [?]96). Patients' diseases and comorbidities were grouped and identified using a widely accepted diagnosis classification system, the International Statistical Classification of Diseases and Related Health Problems, $10^{\text {th }}$ revision (ICD-10). ${ }^{18}$ Each prescription of an individual patient contained the information of the generic names and brand names, duration and route of administration of each medication, as well as the date of the prescription.

\section{PIMs criteria for evaluation}

Each prescription was evaluated based on the updated 2019 Beers Criteria by one of two pharmacists who were familiar with Beers Criteria. According to the 2019 Beers criteria, patients' medical comorbidities were classified into the following diseases or conditions: congestive heart failure, syncope, delirium, insomnia, Parkinson's disease, dementia or cognitive impairment, chronic seizures or epilepsy, history of falls or fractures, history of gastric or duodenal ulcers, and impaired renal function (chronic kidney disease stage four or less).

\section{Statistical analysis}

Logistic regression and univariate analyses were performed to determine the predictors of PIMs in our study population. The candidate predictors included age, sex, number of comorbidities, number of prescribed medications, and classes of healthcare facilities (first-tier, second-tier or third-tier hospitals). Detailed information on the classification of three-tiered healthcare system in China is provided in the online supplementary appendix table 1. Statistical analyses were performed with the application of SPSS Version 19.0 (Statistical Product and Service Solutions) for Windows.

\section{RESULTS}

\section{Demographics of patient and the prevalence of PIMs}


The mean age of the total 4,847,181 patients included in this study was $73.6 \pm 7.6$ years, and $47.9 \%$ $(\mathrm{n}=2,322,541)$ of them were female. Details of patient demographics by sex were described in Table 1. The patients had an average of four comorbidities, among which hypertension $(\mathrm{n}=3,065,235,63.2 \%)$, coronary heart disease $(\mathrm{n}=2,259,965,46.6 \%)$, diabetes mellitus $(\mathrm{n}=1,732,952,35.8 \%)$, hyperlipidemia $(\mathrm{n}=811,402$, $16.7 \%)$, cancer $(\mathrm{n}=570,849,11.8 \%)$ were the five highest-ranked comorbidities.

In total, $40.1 \%$ of elderly patients were prescribed with at least one PIM based on the 2019 Beers Criteria, including $22.3 \%$ prescribed with one PIM and $14.8 \%$ with two. The highest PIM prevalence was observed in male patients of 91 to 95 years group (26.4\%), and in female patients of above 95 years group (20.9\%). In every age group, the percentage of male patients with PIM prescriptions was higher than that of female patients. The prevalence of PIMs by age group and gender in the one-year study period was illustrated in figure 1.

\section{The most common patterns of PIMs}

The average number of medications prescribed was $6.0 \pm 1.5$. The most frequently prescribed PIMs independent of diagnoses or conditions were proton-pump inhibitors $(12.9 \%)$, diuretics $(8.2 \%)$ and central nervous system medications $(6.9 \%)$. The most common category of central nervous system (CNS) medications that were inappropriately used fell into benzodiazepines, predominantly accounting for $76.8 \%$ of all CNS medications. Cardiovascular medications $(2.7 \%)$, aspirin for primary prevention of cardiovascular events in adults aged over $70(2.7 \%)$, pain medications (2.1\%) and drugs with strong anticholinergic properties (2.0\%) respectively took up a small proportion of the PIM prescriptions as well. The prevalence of PIMs independent of diagnoses or conditions was provided in Supplement Table 1.

\section{Drug-Disease and Drug-Syndrome PIMs}

According to the Beers Criteria's lists of drug-disease interactions, elderly patients with central nervous system conditions or with a history of falls or fractures were exposed to PIMs the most. $36.9 \%$ of the patients diagnosed with delirium were prescribed with antipsychotics (21.6\%, olanzapine and quetiapine), followed by benzodiazepines (11.0\%, estazolam, lorazepam, alprazolam etc.). $14.4 \%$ of patients with dementia or cognitive impairment were treated with antipsychotics (9.1\%) and benzodiazepines (5.3\%). $7.1 \%$ of patients with a history of falls or fractures were prescribed with benzodiazepines and antipsychotics. PIMs regarding drug-disease or drug-syndrome interactions were illustrated in Supplement Table 2.

\section{Factors associated with PIM}

PIM related factors were also investigated. Comparing patients with PIM and without PIM, we did not discover a statistically significant difference in neither gender ( $46.5 \%$ in women vs $53.5 \%$ in men) nor age (73.5 \pm 7.3 years in patients with PIM vs $72.6 \pm 6.7$ in patients without PIM, P $>0.05$ for both). However, we found via logistic regression that PIM was associated with age ([?]80 years old), number of conditions ([?]5), number of prescribed medications ([?]10), and hospital tier. Factors associated with PIMs were showed in Table 2.

\section{DISCUSSION}

\section{PIM among Chinese elderly patients}

The problem of PIMs is an important healthcare issue. We discovered through this study that the prevalence of PIMs was $40.1 \%$ in our investigated population. To our knowledge, no study has yet investigated the prevalence of PIM in the whole Chinese elderly population. The results of our study demonstrated and confirmed our proposal that inappropriate medications were frequently being prescribed to senior patients in China, and the prevalence of PIM was slightly higher than what was found based on the 2012 Beers Criteria in Japan (37.5\%), ${ }^{19}$ in the United States $(34.1 \%),{ }^{20}$ in Turkey $(33.3 \%),{ }^{21}$ in Hong Kong $(38.6 \%),{ }^{22}$ and in Albania (34.5\%). ${ }^{23}$ A 2011 Taiwan study revealed a PIM prevalence of $36.2 \%$ based on STOPP/START criteria. $^{24}$ 
The 2019 updated Beers Criteria retained the five categories of criteria in the previous version. The first category includes inappropriate medications for elderly patients. The second and fourth categories are compiled based on drug-disease interactions and drug-drug interactions. The third category comprises medications to be administered with caution on certain situations. The last category takes into account the renal functions of elderly population and suggests an substitute or a dosage reduce of an medication. ${ }^{5}$ Notably, glimepiride has been included to the lists of drug to avoid in elderly patients considering the increased likelihood of prolonged hypoglycemia. ${ }^{5}$ The serotonin-norepinephrine reuptake inhibitors (SNRIs), such as venlafaxine, duloxetine and more, have been advised to be avoided in patients with a history of falls or fractures, where the use of the group of medications could result in future additional falls. ${ }^{5} \mathrm{H}_{2}$-receptor antagonists, e.g. ranitidine, were removed from the "avoid" list in elderly patients with cognitive impairment or dementia. ${ }^{5}$

Beers Criteria are designed for the purpose of warning practitioners of some absolute contraindications for elderly patients, but to be used as an approach to prescribe and monitor adverse effects. The information about the risks of each specific medication should serve to raise practitioners' awareness on the potential problems that may arise with the prescription, but it should be noted that the use of a Beers-Criteria-list drug may still be necessary under some circumstances. Therefore, clinicians should carefully weigh the potential risks and benefits when tailoring medication therapies.

\section{Prescribing benzodiazepines in the elderly}

Benzodiazepines and antipsychotics have been widely known as an indicator that could considerably raise older patients' risk of falls, fractures, and even mortality. ${ }^{25,26}$ However, these medications are commonly prescribed in China due to an insufficient vigilance of adverse outcomes that they might bring about. In the present study, $80.5 \%$ of insomnia patients were taking at least one benzodiazepine, in majority due to the common complaining of sleep disorders.

Suspending the use of benzodiazepines and choosing other alternative drugs that lead to a lower risk of falls could hopefully minimize the possibility of relevant adverse outcomes, which in turn, avoids unnecessary healthcare spending in both resources and expenditures. ${ }^{27}$ Thus, we suggest physicians and pharmacists to pay more attention in the prescribing of benzodiazepines for elderly patients, particularly long-acting benzodiazepines.

\section{Advantage}

One advantage of our study is that we collected large sample data from seventy-six hospitals, therefore our results may credibly reflect current situation of prescription behavior in the higher-level hospitals in China. Second, since we only included inpatients, the uncertainty regarding patients' adherence to dispensed medications could be possibly minimized due to the direct practice and surveillance of healthcare providers.

\section{Limitations}

Limitations still exist and cautions should be taken when interpreting these results. Firstly, Beers Criteria contains some medications that are not currently available on Chinese market, and vice versa; racial difference attributable to enzyme system may result in a change in drug dosage limitations, or even influence the effectiveness of certain drugs. Secondly, non-prescription medications such as over-the-counter drugs or drugs independently purchased by patients from other sources could not be accounted for in the data, which might lead to an underestimation of PIM. Thirdly, the Beers criteria do not include an evaluation of potentially prescribing omissions (PPOs) where safer or more effective alternatives are recommended. PPO is also a crucial issue that should be evaluated simultaneously with PIM when investigating the present prescribing manners.

\section{CONCLUSION}

In conclusion, our results showed that prescription of PIMs is prevalent among Chinese elderly patients hospitalized, which was consistent with other findings of previously published studies. To our knowledge, this was the first study in China to detect inappropriate medications based on Beers Criteria 2019. Application 
of explicit criteria identifying PIMs prescriptions in China is of great urgency, and efforts should be made to optimize prescribing behaviors in clinical settings.

\section{Acknowledgements}

Thanks to pharmacist Yin-Qing Ying of Huashan Hospital, Fudan University, for preparation of the figures.

\section{Contributors}

All authors discussed and commented the results on the manuscript. Ming-Kang Zhong and Ming-Ming Yan designed the study; Ming-Ming Yan, Jing Li and Nang Lu performed the study; Jing Li and Nang Lu contributed production of form and analytic tools; Xiao-Yan Qiu and Shu-Shan Wu analyzed and interpreted of data; Ming-Ming Yan and Shu-Shan Wu drafted the manuscript; Xiao-Yan Qiu and Ming-Ming Yan revised the manuscript; Ming-Kang Zhong and Ming-Ming Yan final approved of the version to be published.

\section{Financial support and sponsorship}

This study was sponsored by Shanghai "Rising Stars of Medical Talent" Youth Development ProgramClinical Pharmacist Program (HWJYZ[2018]1).

\section{Conflicts of interest statement}

There was no conflict of interest that any of the authors should disclose.

\section{REFERENCES}

1. Beers MH. Explicit criteria for determining potentially inappropriate medication use by the elderly: An update. Arch Intern Med . 1997;157(14):1531-1536. doi:10.1001/archinte.1997.00440350031003

2. Fick DM, Cooper JW, Wade WE, Waller JL, Maclean JR, Beers MH. Updating the Beers Criteria for Potentially Inappropriate Medication Use in Older Adults: Results of a US Consensus Panel of Experts. Arch Intern Med . 2003;163(22):2716-2724. doi:10.1001/archinte.163.22.2716

3. Fick DM, Semla TP, Beizer J, et al. American Geriatrics Society updated Beers Criteria for potentially inappropriate medication use in older adults. J Am Geriatr Soc . 2012;60(4):616-631. doi:10.1111/j.15325415.2012.03923.x

4. Fick DM, Semla TP, Beizer J, Brandt N, Dombrowski R. American Geriatrics Society 2015 Updated Beers Criteria for Potentially Inappropriate Medication Use in Older Adults. J Am Geriatr Soc . 2015;63(11):22272246. doi:10.1111/jgs.13702

5. Fick DM, Semla TP, Steinman M, et al. American Geriatrics Society 2019 Updated AGS Beers Criteria for Potentially Inappropriate Medication Use in Older Adults. J Am Geriatr Soc . 2019;67(4):674-694. doi:10.1111/jgs.15767

6. Page RL, Linnebur SA, Bryant LL, Ruscin JM. Inappropriate prescribing in the hospitalized elderly patient: Defining the problem, evaluation tools, and possible solutions. Clin Interv Aging . 2010;5:75. doi:10.2147/cia.s9564

7. Stuck AE, Beers MH, Steiner A, Aronow HU, Rubenstein LZ, Beck JC. Inappropriate Medication Use in Community-Residing Older Persons.Arch Intern Med . 1994;154(19):2195-2200. doi:10.1001/archinte.1994.00420190095011

8. Jhaveri BN, Patel TK, Barvaliya MJ, Tripathi C. Utilization of potentially inappropriate medications in elderly patients in a tertiary care teaching hospital in India. Perspect Clin Res . 2014;5(4):184-189. doi:http://dx.doi.org/10.4103/2229-3485.140562

9. Bahat G, Tufan F, Bahat Z, et al. Comorbidities, polypharmacy, functionality and nutritional status in Turkish community-dwelling female elderly. Aging Clin Exp Res . 2014;26(3):255-259. doi:10.1007/s40520014-0229-8 
10. Stockl KM, Le L, Zhang S, Harada AS. Clinical and economic outcomes associated with potentially inappropriate prescribing in the elderly. Am J Manag Care . 2010;16(1):e1-10.

11. Fick DM, Mion LC, Beers MH, Waller JL. Health outcomes associated with potentially inappropriate medication use in older adults. Res Nurs Heal . 2008;31(1):42-51. doi:10.1002/nur.20232

12. Keith SW, Maio V, Dudash K, Templin M, Del Canale S. A physician-focused intervention to reduce potentially inappropriate medication prescribing in older people: A 3-year, Italian, prospective, proof-ofconcept study. Drugs and Aging . 2013;30:119-127. doi:10.1007/s40266-012-0043-y

13. Gupta S, Rappaport HM, Bennett LT. Inappropriate drug prescribing and related outcomes for elderly Medicaid beneficiaries residing in nursing homes. Clin Ther . 1996;18(1):183-196. doi:10.1016/S01492918(96)80189-5

14. Kaur S, Mitchell G, Vitetta L, Roberts MS, Gallagher P. Interventions that can Reduce Inappropriate Prescribing in the Elderly: A Systematic Review. Drugs and Aging . 2009;26(12):1013-1028. doi:10.2165/11318890-000000000-00000

15. Rankin A, Cadogan C, Patterson S, et al. Interventions to improve the appropriate use of polypharmacy for older people. Cochrane Database Syst Rev . 2018;(9). doi:10.1002/14651858.CD008165.pub4

16. Tannenbaum C, Martin P, Tamblyn R, Benedetti A, Ahmed S. Reduction of inappropriate benzodiazepine prescriptions among older adults through direct patient education: The EMPOWER cluster randomized trial.JAMA Intern Med . 2014;174(6):890-898. doi:10.1001/jamainternmed.2014.949

17. Yan MM, Wu SS, Ying YQ, Lu N, Zhong MK. Safety assessment of concurrent statin treatment and evaluation of drug interactions in China. SAGE Open Med . 2018;6:1-9. doi:10.1177/2050312118798278

18. World Health Organization. International Statistical Classification of Diseases and Related Health Problems, 10th Revision (ICD-10) .; 2016.

19. Ishii S, Kojima T, Ezawa K, et al. The association of change in medication regimen and use of inappropriate medication based on beers criteria with adverse outcomes in Japanese long-term care facilities. Geriatr Gerontol Int . 2017;17:591-597. doi:10.1111/ggi.12761

20. Brown JD, Hutchison LC, Li C, Painter JT, Martin BC. Predictive Validity of the Beers and Screening Tool of Older Persons' Potentially Inappropriate Prescriptions (STOPP) Criteria to Detect Adverse Drug Events, Hospitalizations, and Emergency Department Visits in the United States. J Am Geriatr Soc . 2016;64(1):22-30. doi:10.1111/jgs.13884

21. Bahat G, Bay I, Tufan A, Tufan F, Kilic C, Karan MA. Prevalence of potentially inappropriate prescribing among older adults: A comparison of the Beers 2012 and Screening Tool of Older Person's Prescriptions criteria version 2. Geriatr Gerontol Int . 2017;17(9):1245-1251. doi:10.1111/ggi.12850

22. Lam MPS, Cheung BMY, Wong ICK. Prevalence of Potentially Inappropriate Prescribing Among Hong Kong Older Adults: A Comparison of the Beers 2003, Beers 2012, and Screening Tool of Older Person's Prescriptions and Screening Tool to Alert doctors to Right Treatment Criteria. J Am Geriatr Soc . 2015;63(7):1471-1472. doi:10.1111/jgs.13555

23. Hudhra K, Beci E, Petrela E, Xhafaj D, Garcia-Caballos M, Bueno-Cavanillas A. Prevalence and factors associated with potentially inappropriate prescriptions among older patients at hospital discharge.J Eval Clin Pract . 2016;22(5):707-713. doi:10.1111/jep.12521

24. Liu CL, Peng LN, Chen YT, Lin MH, Liu LK, Chen LK. Potentially inappropriate prescribing (IP) for elderly medical inpatients in Taiwan: A hospital-based study. Arch Gerontol Geriatr . 2012;55(1):148-151. doi:10.1016/j.archger.2011.07.001

25. Herings RMC, Stricker BHC, Boer A, Bakker A, Sturmans F. Benzodiazepines and the Risk of Falling Leading to Femur Fractures: Dosage More Important Than Elimination Half-life. Arch Intern Med . 
1995;155(16):1801-1807. doi:10.1001/archinte.1995.00430160149015

26. Wang PS, Schneeweiss S, Avorn J, et al. Risk of Death in Elderly Users of Conventional vs. Atypical Antipsychotic Medications. N Engl J Med . 2005;353(22):2335-2341. doi:10.1056/nejmoa052827

27. Panneman MJM, Goettsch WG, Kramarz P, Herings RMC. The costs of benzodiazepine-associated hospital-treated fall injuries in the EU: A pharmo study. Drugs and Aging . 2003;20(11):833-839. doi:10.2165/00002512-200320110-00004

Figure 1. One-year period prevalence of PIMs in terms of age groups and sex in 2017.

Figure 1

Yan et al

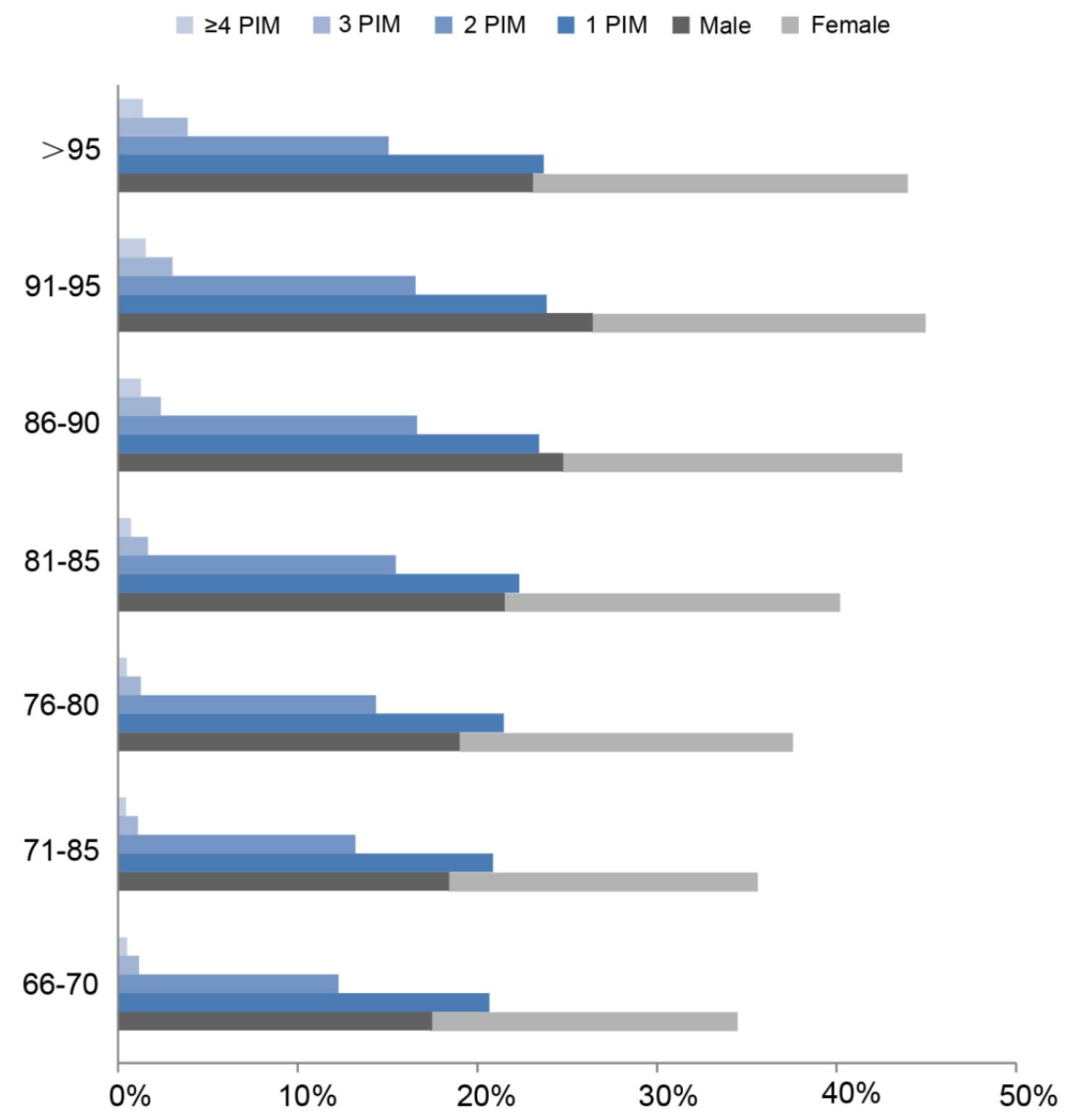


Figure 1

Yan et al

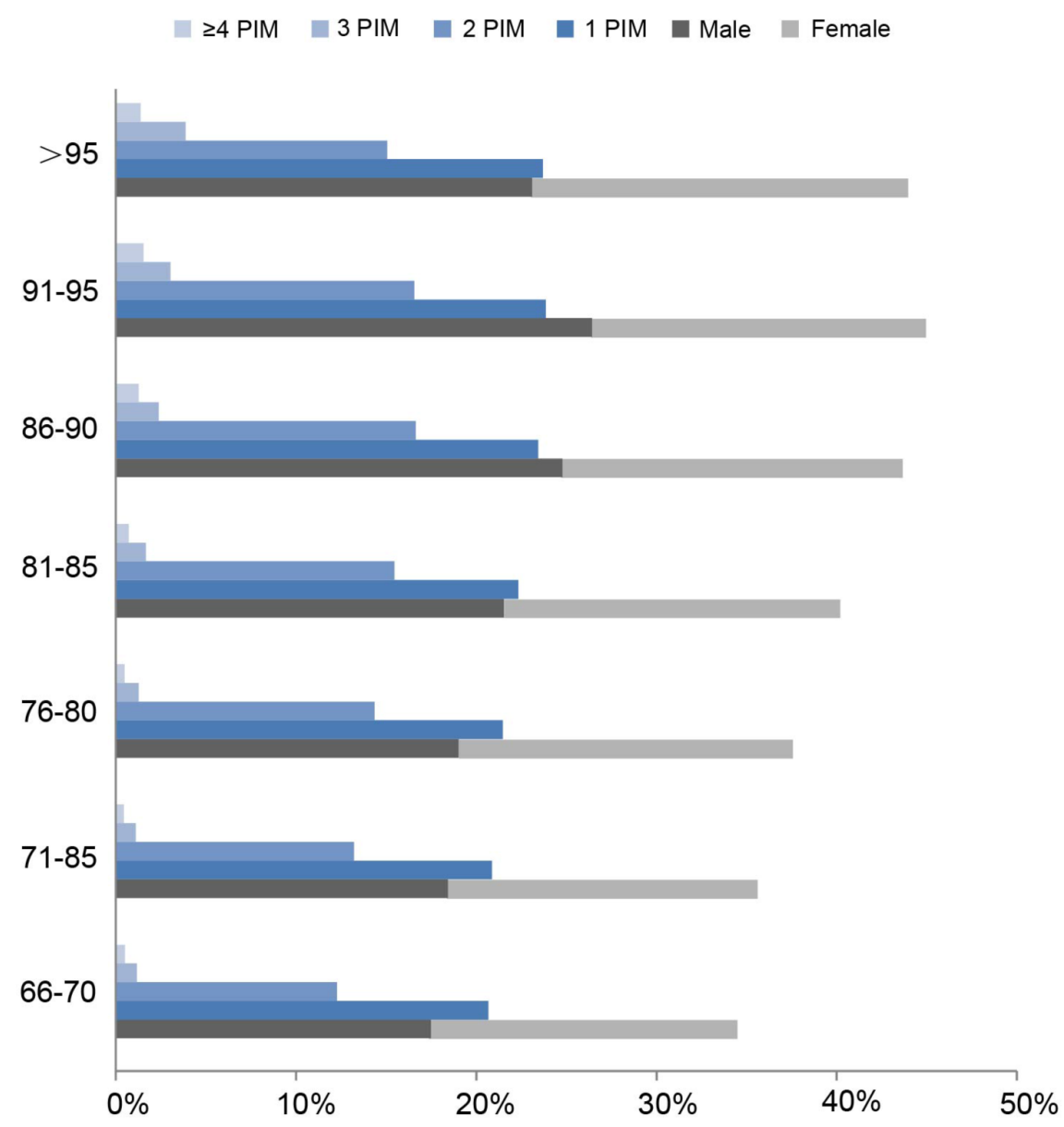

\section{Hosted file}

Table 1.docx available at https://authorea.com/users/327773/articles/455214-factorsassociated-with-potentially-inappropriate-medication-use-in-older-adults-in-chinaapplying-2019-updated-beers-criteria

\section{Hosted file}

Table 2.docx available at https://authorea.com/users/327773/articles/455214-factorsassociated-with-potentially-inappropriate-medication-use-in-older-adults-in-chinaapplying-2019-updated-beers-criteria

\section{Hosted file}

Supplement Table 1.docx available at https://authorea.com/users/327773/articles/455214factors-associated-with-potentially-inappropriate-medication-use-in-older-adults-inchina-applying-2019-updated-beers-criteria

\section{Hosted file}


Supplement Table 2.docx available at https://authorea.com/users/327773/articles/455214factors-associated-with-potentially-inappropriate-medication-use-in-older-adults-inchina-applying-2019-updated-beers-criteria

\section{Hosted file}

Supplementary Appendix 1.docx available at https://authorea.com/users/327773/articles/455214factors-associated-with-potentially-inappropriate-medication-use-in-older-adults-inchina-applying-2019-updated-beers-criteria 